\title{
COMPARISON OF ANTIHYPERGLYCEMIC ACTIVITY OF DIFFERENT PARTS OF KLUTUK BANANA (MUSA BALBISIANA COLLA)
}

\author{
TITA NOFIANTI1 ${ }^{13}$, AHMAD MUHTADI ${ }^{*}$, IRDA FIDRIANNY²
}

${ }^{1}$ Department of Pharmacology, Faculty of Pharmacy, Padjadjaran University, Bandung, Indonesia, ${ }^{2}$ Department of Pharmaceutical Biology, School of Pharmacy, Bandung Institute of Technology, Indonesia, ${ }^{3}$ Department of Pharmacology, Institute of Health Science of Bakti Tunas Husada Tasikmalaya, West Java, Indonesia Email: a.muhtadi@unpad.ac.id

Received: 27 Sep 2020, Revised and Accepted: 18 Oct 2020

\section{ABSTRACT}

Objective: Banana is a plant that grows in Indonesia and is widely consumed by Indonesian people. One of banana type is klutuk banana (Musa balbisiana Colla). There were many types of researches about klutuk banana for antidiabetic activity; however, antidiabetic activity of its peel and pulp are still unknown.

Methods: The objective of the study was to determine of antihyperglycemic activity of different parts of klutuk banana. Animals were divided into 15 groups, namely normal control, negative control, positive control (glibenclamide $0.65 \mathrm{mg} / \mathrm{kg}$ body weight (bw), and 12 sample groups. All animals were given $2 \mathrm{~g} / \mathrm{kg}$ bw glucose monohydrate and blood glucose level was measured every $30 \mathrm{~min}$ for $120 \mathrm{~min}$.

Results: The results of the oral glucose tolerance test (OGTT) showed that KPE2 (klutuk peel extract $350 \mathrm{mg} / \mathrm{kg}$ bw) gave higher activity to decrease blood glucose level compared to the other groups at the minute of $30(-24.83 \% ;$ p. 0.00$), 60(-33.93 \% ;$ p. 0.000$), 90(-46.29 \%$; p. 0.000$)$ and $120(-35.44 \%$; p. 0.000)

Conclusion: The klutuk peel extract has very strong antioxidant activity and antihyperglycemic activity at a dose of $350 \mathrm{mg} / \mathrm{kg}$ bw.

Keywords: Antihyperglycemic, Musa balbisiana, Antioxidant, Different parts, OGTT

(C) 2021 The Authors.Published by Innovare Academic Sciences Pvt Ltd. This is an open access article under the CCBYlicense (https://creativecommons.org/licenses/by/4.0/) DOI: https://dx.doi.org/10.22159/ijap.2021.v13s3.12 Journal homepage: https://innovareacademics.in/journals/index.php/ijap

\section{INTRODUCTION}

Current development has changed human beings in all aspects, such as lifestyle, which present known as the term of 3F: fun, fashion and food [1]. These changes impact on changes in the pattern of diseases from infectious towards degenerative diseases such as diabetes mellitus (DM). Especially for developing countries, including Indonesia, which resulted in changes both in lifestyle and diet, which one was the risk factors for DM [2].

According to the International Diabetic Federation (IDF), it is estimated that almost half (49.7\%) of all people were living with undiagnosed diabetes in 2017. In addition, about 374 million people have glucose tolerance impaired and it was projected about 21.3 million birth affected by hyperglycemia in pregnancy. In 2017, about 5 million deaths around the world were caused by diabetes. Indonesia ranked 6th in DM and affected 10.3 million people after China, India, United States, Brazil and Mexico [3, 4]. According to data Basic Health Research of the Ministry of Health of Indonesia, DM prevalence increased from 6.9\% (2013) to 8.5\% (2018). Diabetes mellitus become a burden in cost due to its needed long-life treatment [5].

Therefore, necessary to find other alternative medicine from nature which claimed less adverse reactions. Klutuk banana (M. balbisiana Colla) is a herbal medicine that grows in Indonesia and is mostly consumed by the Indonesian people. This plant has anti-diabetic, anti hyperlipidemia, antioxidant and antibacterial activity [6-9]. Bananas have been classified as one of the rich antioxidant foods [10]. This antioxidant activity has a good role for diabetes mellitus improvement through increase of catalase, decrease of malondialdehyde and increase of insulin level. As known hyperglycemia lead to oxidative stress production and destruct the pancreas [7]. Previous studies showed that the ethanolic extract of flower and stem of M. balbisiana Colla a dose of $250 \mathrm{mg} / \mathrm{kg}$ bw inhibited the absorption of glucose in the intestines [6, 7]. However, antihyperglycemic activity of peel and pulp of klutuk banana are still unknown.

\section{MATERIALS AND METHODS}

\section{Materials}

D-glucose, alloxan, 2,2-diphenyl-1-picrylhydrazyl (DPPH) and sodium carboxymethyl cellulose (CMC-Na) were purchased from
Sigma Aldrich, chemistry and biotechnology Inc. (St. Louis, Missouri United States). Glibenclamide was purchased from Kimia Farma, Indonesia. Strips test for blood glucose examination was purchased from All Medicus Co., Ltd. The Republic of Korea. Methanol and ethanol analytical grade were purchased from Merck.

\section{Methods}

Preparation of plant material

All parts of klutuk banana ( $M$. balbisiana Colla) peel, pulp, seed and flower were obtained from November 2018 at Tasikmalaya, West Java, Indonesia. The identity of the plant was confirmed by the Faculty of Mathematics and Natural Sciences; Departement of Biology, Padjadjaran University, with numbers: 156/HB/11/2018. All parts were washed, dried, cut into pieces, then ground. The powder was stored at a dry place for further use.

\section{Preparation of extract}

All parts of klutuk banana ( $M$. balbisiana Colla) were extracted by reflux method with $96 \%$ ethanol for $3 \mathrm{~h}$ and repeated three times. Furthermore, the sample was filtered through filter paper and then vaporated by the rotary evaporator. This extract was kept in the refrigerator at $4^{\circ} \mathrm{C}$ for further use.

\section{Phytochemical screening}

The phytochemical screening was performed to detect the presence of secondary metabolites such as alkaloid, flavonoid, saponin, steroid/triterpenoid, tannin, quinone, and phenols. Phytochemical screening was performed on crude drug and extracts of different parts of klutuk banana (M. balbisiana Colla) [7].

\section{Preparation of animals}

Adult male mice 2-3 mo (23-28 g) were obtained from the Experimental Animal Application and Research Center of Biofarma. The animals were kept at a constant temperature $\left(24 \pm 2{ }^{\circ} \mathrm{C}\right)$ with $50 \%$ humidity, 12-h light-dark cycles and were allowed ad libitum access to standard mice chow and water. The animals were adapted in one week and divide in 15 groups, 12 treatment groups and 3 
control groups. Number of animals in each group was calculated using Federer equation. The study was approved by Research Ethics Committee, Padjadjaran University (KEPK UNPAD) with no. 330/UN6. KeP/EC/2019.

\section{Experimental design}

There were 15 groups and each group contained 5 animals. Normal (CMC-Na 0.5\%); negative control (CMC-Na 0.5\%); positive control (glibenclamide $0.65 \mathrm{mg} / \mathrm{kg}$ bw); 12 sample groups KPE1 (ethanolic klutuk peel extract $175 \mathrm{mg} / \mathrm{kg}$ bw), KPE2 (350 mg/kg bw), KPE3 (700 mg/kg bw), KPU1 (ethanolic klutuk pulp extract $175 \mathrm{mg} / \mathrm{kg}$ bw), KPU2 (350 mg/kg bw), KPU3 (700 mg/kg bw), KSE1 (ethanolic klutuk seed extract $175 \mathrm{mg} / \mathrm{kg}$ bw), KSE2 (350 mg/kg bw), KSE3 (700 mg/kg bw), KFL1 (ethanolic klutuk flower extract $175 \mathrm{mg} / \mathrm{kg}$ bw), KFL2 (350 mg/kg bw), and KFL3 (700 mg/kg bw).

\section{Antidiabetic activity test oral glucose tolerance test (OGTT) method}

The oral glucose tolerance test (OGTT) was applied to evaluate the ability of glucose metabolism regulation, glucose-induced insulin secretion, and glycemic changes. After fasting $12 \mathrm{~h}$ previously, all mice were given different treatments depending on the groups, then after 30 min, all groups were orally administered of glucose $(2 \mathrm{~g} / \mathrm{kg} \mathrm{bw})$. The glucose concentration was measured from the collected blood tail vein from at 0 (just before glucose administration), 30, 60, 90, and 120-min using glucose oxidase-peroxidase reactive strips and the glucometer (Gluco-Dr Biosensor AGM 2100, All Medicus Co., Ltd, Korea) [12].

\section{Antidiabetic activity test alloxan-induced method}

Mice were induced using an intraperitoneal injection of alloxan monohydrate $(170 \mathrm{mg} / \mathrm{kg}$ bw) (Sigma, Germany) which was dissolved in normal saline immediately before used [13, 14]. Diabetes was verified in the animal by measuring fasting blood glucose $3 \mathrm{~d}$ following alloxan injection. The rats with glucose level more than $200 \mathrm{mg} / \mathrm{dL}$ were used for the study $[12,13]$.

\section{Antioxidant activity test}

The antioxidant activity was carried out for ethanol peel extracts of klutuk Musa balbisiana Colla, which was assessed by calculating the scavenging effect of free radical DPPH. The diluted test solutions of peel extracts were prepared in methanol. DPPH $30 \mu \mathrm{g} / \mathrm{ml}$ was prepared in methanol and $1 \mathrm{ml}$ of this solution was mixed with $1 \mathrm{ml}$ of sample solutions. These mixtures were kept in the dark for $30 \mathrm{~min}$ and absorbance was measured at $\lambda 516 \mathrm{~nm}$ using a UV-Visible spectrophotometer. DPPH solution was used as control [14]. The absorbance was recorded and \% inhibition was calculated. IC50 value can be investigated utilizing a calibration curve. The Antioxidant Activity Index (AAI) was determined by dividing final concentration of DPPH with IC50 value.

\section{Statistical analysis}

All data were expressed as mean $\pm S D$. The SPSS package (version 16) was applied in statistical analysis. The comparison between groups was performed by one-way ANOVA, followed by post hoc Tukey $[15,16]$.

\section{RESULTS AND DISCUSSION}

Extraction of each part of klutuk banana was carried out by reflux using $96 \%$ ethanol. The yield and density of extracts is provided in table 1. The extract was concentrated using a vacuum rotary evaporator. The yield extracts of the present research were $4.31 \%$, $8.18 \%, 5.10 \%$, and $4.63 \%$ for flower, peel, pulp and seed, respectively. Meanwhile, in research by Borah and Das, which used the percolation method using $95 \%$ ethanol solvent, the yields extracts were: flower $12.17 \%$ and stalk $10.57 \%$ [7]. While, the other study stated that the yield of seed extracts was: hexane $0.66 \%$, acetone $3.87 \%$, ethanol $0.55 \%$ and aqueous extract $1.0 \%$ [17].

Table 1: Yield and density of extracts

\begin{tabular}{lll}
\hline Sample & Yield extract (\%) & Density 1\% extract (g/ml) \\
\hline Peels & 8.18 & $0.83 \pm 0.005$ \\
Pulps & 5.10 & $0.82 \pm 0.006$ \\
Seeds & 4.63 & $0.80 \pm 0.002$ \\
Flowers & 4.31 & $0.82 \pm 0.005$ \\
\hline
\end{tabular}

Phytochemical screening was carried out on crude drug and extract of flower, peel, pulp and seed of klutuk banana (M. balbisiana Colla). The results showed that flower, peel, pulp and seed positive for flavonoid, phenols and steroid/triterpenoid (table 2). Saponin was detected only in peel, pulp and seed. In while, tannin was detected only in the flower and peel.

Table 2: Phytochemical screening of crude drug and extract of $M$. balbisiana

\begin{tabular}{lllllllll}
\hline Groups & Peels & & Pulps & & Seeds & & \multicolumn{2}{l}{ Flowers } \\
\cline { 2 - 8 } & Crude drug & Extract & Crude drug & Extract & Crude drug & Extract & Crude drug & Extract \\
\hline Alkaloids & $(-)$ & $(-)$ & $(-)$ & $(-)$ & $(-)$ & $(-)$ & $(-)$ & $(-)$ \\
Flavonoids & $(+)$ & $(+)$ & $(+)$ & $(+)$ & $(+)$ & $(+)$ & $(+)$ & $(+)$ \\
Phenols & $(+)$ & $(+)$ & $(+)$ & $(+)$ & $(+)$ & $(+)$ & $(+)$ & $(+)$ \\
Tannins & $(+)$ & $(+)$ & $(-)$ & $(-)$ & $(-)$ & $(-)$ & $(+)$ & $(+)$ \\
Saponins & $(+)$ & $(+)$ & $(+)$ & $(+)$ & $(+)$ & $(+)$ & $(-)$ & $(-)$ \\
Quinones & $(-)$ & $(-)$ & $(+)$ & $(-)$ & $(-)$ & $(-)$ & $(-)$ & $(-)$ \\
Steroids/triterpenoids & $(+)$ & $(+)$ & $(+)$ & $(+)$ & $(+)$ & $(+)$ & $(+)$ & $(+)$ \\
\hline
\end{tabular}

$(+)$ identified, $(-)$ not identified

The bananas have been known had antidiabetic activities [17-19]. The active compounds of bananas included lupeol, ferulic acid, vanillic acid, trans-cinnamic acid, $\mathrm{p}$-hydroxybenzoic acid, $\mathrm{p}$ coumarate acid, rutin, catechin/epicatechin, chlorogenic acid, gallic acid, caffeic acid, nicotiflorine [20]. Furthermore, flower and stalk of M. balbisiana Colla also had antihyperlipidemic, antioxidants and antidiabetic activity by streptozotocin (STZ)-induced type 1 diabetic rats $[17,18,21]$. The active secondary metabolites which were found in these parts such as flavonoids, tannins, saponins, diterpenes, triterpenes, and phenols $[7,8]$.

The results of antihyperglycemic effect of different parts of klutuk banana (M. balbisiana Colla) were presented in table 3 . The percentage of blood glucose level reduction was demonstrated in fig. 1. The parts of M. balbisiana Colla which showed higher antihyperglycemic effect through OGTT method, then continued by using alloxan induced method (table 4). 


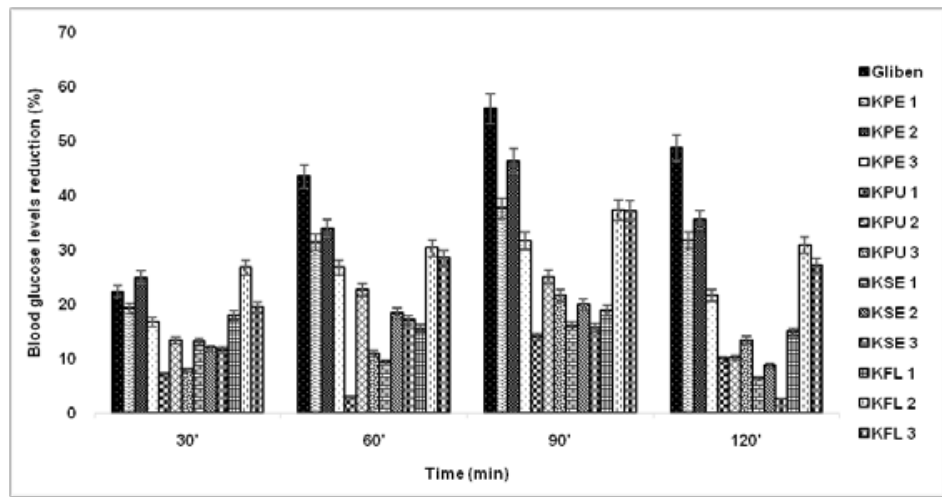

Fig. 1: The reduction percentage of blood glucose levels after treatment by different parts extracts of M. balbisiana Colla

The glucose tolerance test method was a preliminary test to determine the ability of the ethanolic extracts of different parts of klutuk banana (M. balbisiana Colla). The percentage blood glucose reduction at $30 \mathrm{~min}$ showed that KFL2 $>$ KPE2> KPL3> KPE $1>$ KFL $1>$ KPE $3>$ KPU2 $>$ KSE $1>$ KSE $2>$ KSE $3>$ KPU $3>$ KPU1. KPE $1,2,3$ and KFL 1,2,3 showed significant different compared to the negative group $(\mathrm{P}<0.01)$. In a while, percentage of blood glucose reduction at $60 \mathrm{~min}$ showed that KPE2 $>$ KPE1 $>$ KFL2 $>$

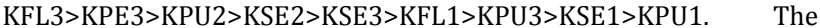
results demonstrated that KPE 1,2,3; KPU2; KSE 2,3 and KFL 1,2,3 figured significantly different compared to negative control $(\mathrm{P}<0.01)$. At 90 min presented that KPE2 $46.29 \%$ had the highest blood glucose level reduction, followed by KPE1 $37.63 \%$ and KPE3 $31.65 \%$. These results demonstrated that KPE1,2,3; KPU1,2,3; KSE1,2,3 and KFL 1,2,3 displayed significant different compared to negative control $(\mathrm{P}<0.01)$. At $120 \mathrm{~min}$ had that the highest blood glucose level reduction was given by KPE2 $35.44 \%$, followed by KPE1 $31.75 \%$. The KPE1,2,3, and KFL 1,2,3 revealed significant different compared to negative control $(\mathrm{P}<0.01)$. These result showed that the highest percentage of blood glucose reduction was given by KPE2 and it was in line with Borah and Das study [7],
Gopalan et al. [17] and Jannat et. al [22] which showed that the peels, flowers and seeds of M. balbisiana and Musa seminifera provided antidiabetic activity. The present study showed KPE2 had the best blood glucose reduction activity at $30 \mathrm{~min}(-24.83 \%$; p. 0.00), $60 \mathrm{~min}$ $(-33.93 \%$; p. 0.000$), 90 \mathrm{~min}(-46.29 \%$; p. 0,000$)$ and $120 \mathrm{~min}(-$ $35.44 \%$; p. 0,000) compared to the negative control.

This result similar to research by Genatrika et al., which provided that ethanol peel extract of Musa acuminata Colla with dose of 500 $\mathrm{mg} / \mathrm{kg}$ bw could reduce blood glucose level $42.62 \%, 375 \mathrm{mg} / \mathrm{kg}$ bw $37.26 \%$ and $250 \mathrm{mg} / \mathrm{kg}$ bw $24.12 \%$ [23]. Research by Jannat et al. expressed that ripe fruit peels of M. seminifera Lour with a dose of $50 \mathrm{mg} / \mathrm{kg}$ bw could reduce blood glucose level $16.5 \%, 100 \mathrm{mg} / \mathrm{kg}$ bw $25.2 \%, 200 \mathrm{mg} / \mathrm{kg}$ bw $29.8 \%$ and $400 \mathrm{mg} / \mathrm{kg}$ bw $35.1 \%$ [24]. AlMahamud et al. studied regarding various sub-clutivar of Musa sapientum, which revealed that the higher dose $(400 \mathrm{mg} / \mathrm{kg} \mathrm{bw})$ of methanol extract gave the higher percentage of decreasing in blood glucose level. Zin sub-cultivar $400 \mathrm{mg} / \mathrm{kg}$ bw $42.9 \%$ showed higher percentage of decreasing in blood glucose level than Bangla subcultivar $400 \mathrm{mg} / \mathrm{kg}$ bw $35.3 \%$ and Champa sub-cultivar $400 \mathrm{mg} / \mathrm{kg}$ bw $34.6 \%$ [24].

Table 3: Antihyperglycemic effect of different parts extracts of $M$. balbisiana by oral glucose tolerance test method

\begin{tabular}{|c|c|c|c|c|c|c|c|}
\hline \multirow[t]{2}{*}{ Groups } & \multicolumn{6}{|c|}{ Blood glucose (mg/dl) } & \multirow{2}{*}{$\begin{array}{l}\text { Blood glucose } \\
\text { reduction }(\%)\end{array}$} \\
\hline & 0 min & $30 \mathrm{~min}$ & $60 \mathrm{~min}$ & $90 \mathrm{~min}$ & $120 \mathrm{~min}$ & Average $120 \mathrm{~min}$ & \\
\hline Normal Control & $95.2 \pm 5.6$ & $90.4 \pm 5.6$ bc & $87.8 \pm 9.5 \mathrm{~b}$ & $86.2 \pm 6.8 \mathrm{~b}$ & $85.6 \pm 9.8 \mathrm{~b}$ & $87.5 \pm 6.3 \mathrm{abc}$ & $3.2 \pm 4.6$ \\
\hline $\begin{array}{l}\text { Negative Control }(2 \mathrm{~g} / \mathrm{kg} \\
\text { bw glucose monohydrate) }\end{array}$ & $99.8 \pm .4 .4$ & $\begin{array}{l}209.4 \pm 9.2 \\
\text { ac }\end{array}$ & $202.8 \pm 7.9$ ac & $\begin{array}{l}194.0 \pm 12.5 \\
\text { ac }\end{array}$ & $\begin{array}{l}151.8 \pm 12.3 \\
\text { ac }\end{array}$ & $189.5 \pm 5.5 \mathrm{ac}$ & $9.5 \pm 3.3$ \\
\hline $\begin{array}{l}\text { Positive Control ( } 0.65 \\
\mathrm{mg} / \mathrm{kg} \text { bw glibenclamide) }\end{array}$ & $99.6 \pm 7.0$ & $\begin{array}{l}162.6 \pm 11.6 \\
\mathrm{ab}\end{array}$ & $114.6 \pm 10.7 \mathrm{~b}$ & $85.6 \pm 10.2 \mathrm{~b}$ & $77.8 \pm 14.3 \mathrm{~b}$ & $110.2 \pm 7.1 \mathrm{ab}$ & $32,3 \pm 5,3$ \\
\hline $\begin{array}{l}\text { KPE1 Klutuk peels } \\
\text { extract(175 mg/kgbw) }\end{array}$ & $96.2 \pm 6.1$ & $\begin{array}{l}169.0 \pm 15.4 \\
\mathrm{ab}\end{array}$ & $\begin{array}{l}139.2 \pm 14.4 \\
\mathrm{ab}\end{array}$ & $\begin{array}{l}121.0 \pm 11.8 \\
\text { abc }\end{array}$ & $\begin{array}{l}103.6 \pm 6.6 \\
\text { bc }\end{array}$ & $133.2 \pm 7.0 \mathrm{abc}$ & $21.2 \pm 4.3$ \\
\hline $\begin{array}{l}\text { KPE2 Klutuk peels extract } \\
(350 \mathrm{mg} / \mathrm{kg} \mathrm{bw})\end{array}$ & $99.4 \pm 4.9$ & $\begin{array}{l}157.4 \pm 9.2 \\
\mathrm{ab}\end{array}$ & $134.0 \pm 9.1 \mathrm{ab}$ & $104.2 \pm 7.3 \mathrm{~b}$ & $98 \pm 4.6 \mathrm{~b}$ & $123.4 \pm 3.9 \mathrm{ab}$ & $21.6 \pm 3.6$ \\
\hline $\begin{array}{l}\text { KPE3 Klutuk peels extract } \\
(700 \mathrm{mg} / \mathrm{kg} \mathrm{bw})\end{array}$ & $96.4 \pm 8.1$ & $\begin{array}{l}174.4 \pm .8 .6 \\
\mathrm{ab}\end{array}$ & $\begin{array}{l}148.4 \pm 10.3 \\
\text { abc }\end{array}$ & $\begin{array}{l}132.6 \pm 6.9 \\
\text { abc }\end{array}$ & $\begin{array}{l}119.0 \pm 12.9 \\
\text { abc }\end{array}$ & $143.6 \pm 4.8 \mathrm{abc}$ & $17.7 \pm 4.6$ \\
\hline $\begin{array}{l}\text { KPU1 Klutuk pulps extract } \\
(175 \mathrm{mg} / \mathrm{kg} \mathrm{bw})\end{array}$ & $99.4 \pm 5.0$ & $\begin{array}{l}194.6 \pm 11.5 \\
\text { ac }\end{array}$ & $197.0 \pm 9.7 \mathrm{ac}$ & $\begin{array}{l}166.8 \pm 11.7 \\
\text { ac }\end{array}$ & $\begin{array}{l}136.8 \pm 11.4 \\
\text { ac }\end{array}$ & $173.8 \pm 3.4 \mathrm{ac}$ & $10.7 \pm 5.4$ \\
\hline $\begin{array}{l}\text { KPU2 Klutuk pulps extract } \\
(350 \mathrm{mg} / \mathrm{kg} \mathrm{bw})\end{array}$ & $\begin{array}{l}100.6 \pm 5 \\
5\end{array}$ & $\begin{array}{l}181.4 \pm 11.9 \\
\mathrm{a}\end{array}$ & $\begin{array}{l}157.0 \pm 10.2 \\
\text { abc }\end{array}$ & $\begin{array}{l}145.4 \pm 13.5 \\
\text { abc }\end{array}$ & $\begin{array}{l}136.4 \pm 13.7 \\
\text { ac }\end{array}$ & $155.1 \pm 4.5 \mathrm{abc}$ & $14.5 \pm 6.2$ \\
\hline $\begin{array}{l}\text { KPU3 Klutuk pulps extract } \\
(700 \mathrm{mg} / \mathrm{kg} \mathrm{bw})\end{array}$ & $\begin{array}{l}101.2 \pm 4 \\
9\end{array}$ & $\begin{array}{l}192.8 \pm 10.8 \\
\text { ac }\end{array}$ & $\begin{array}{l}180.8 \pm 13.8 \\
\text { ac }\end{array}$ & $\begin{array}{l}152.0 \pm 15.1 \\
\text { abc }\end{array}$ & $\begin{array}{l}131.4 \pm 12.5 \\
\text { ac }\end{array}$ & $164.3 \pm 8.6 \mathrm{abc}$ & $14.8 \pm 3.5$ \\
\hline $\begin{array}{l}\text { KSE1 Klutuk seeds extract } \\
\text { (175 mg/kg bw) }\end{array}$ & $99.4 \pm 4.6$ & $\begin{array}{l}181.8 \pm 13.4 \\
\mathrm{a}\end{array}$ & $184 \pm 12.3 \mathrm{ac}$ & $\begin{array}{l}162.8 \pm 13.0 \\
\text { abc }\end{array}$ & $\begin{array}{l}142.2 \pm 17.0 \\
\text { ac }\end{array}$ & $167.7 \pm 11.1 \mathrm{abc}$ & $7.7 \pm 5.3$ \\
\hline $\begin{array}{l}\text { KSE2 Klutuk seeds extract } \\
(350 \mathrm{mg} / \mathrm{kg} \mathrm{bw})\end{array}$ & $92.6 \pm 8.8$ & $\begin{array}{l}184.6 \pm 15.8 \\
\mathrm{a}\end{array}$ & $\begin{array}{l}165.6 \pm 11.3 \\
\text { abc }\end{array}$ & $\begin{array}{l}155.2 \pm 14.9 \\
\text { abc }\end{array}$ & $\begin{array}{l}138.6 \pm 11.6 \\
\text { ac }\end{array}$ & $161.0 \pm 6.7 \mathrm{abc}$ & $12.8 \pm 5.6$ \\
\hline $\begin{array}{l}\text { KSE3 Klutuk seeds extract } \\
(700 \mathrm{mg} / \mathrm{kg} \mathrm{bw})\end{array}$ & $\begin{array}{l}100.2 \pm 8 \\
8\end{array}$ & $185.2 \pm 8.0 \mathrm{a}$ & $\begin{array}{l}168.2 \pm 13.7 \\
\text { abc }\end{array}$ & $\begin{array}{l}163.6 \pm 12.6 \\
\text { ac }\end{array}$ & $\begin{array}{l}147.8 \pm 11.6 \\
\text { ac }\end{array}$ & $166.2 \pm 7.9 \mathrm{abc}$ & $10.3 \pm 3.6$ \\
\hline $\begin{array}{l}\text { KFL1 Klutuk flowers } \\
\text { extract }(175 \mathrm{mg} / \mathrm{kg} \mathrm{bw})\end{array}$ & $99.4 \pm 2.4$ & $\begin{array}{l}171.8 \pm 12.1 \\
\mathrm{ab}\end{array}$ & $\begin{array}{l}171.2 \pm 10.7 \\
\text { abc }\end{array}$ & $\begin{array}{l}157.4 \pm 10.3 \\
\text { abc }\end{array}$ & $\begin{array}{l}129.2 \pm 13.7 \\
\text { ac }\end{array}$ & $157.4 \pm 4.3 \mathrm{abc}$ & $8.4 \pm 5.7$ \\
\hline $\begin{array}{l}\text { KFL2 Klutuk flowers } \\
\text { extract }(350 \mathrm{mg} / \mathrm{kg} \mathrm{bw})\end{array}$ & $99.2 \pm 5.4$ & $\begin{array}{l}153.4 \pm 12.5 \\
\mathrm{ab}\end{array}$ & $\begin{array}{l}141.2 \pm 10.1 \\
\mathrm{ab}\end{array}$ & $\begin{array}{l}121.8 \pm 12.6 \\
\text { abc }\end{array}$ & $105.0 \pm 5.7 \mathrm{~b}$ & $130.4 \pm 6.4 \mathrm{abc}$ & $15.1 \pm 3.4$ \\
\hline $\begin{array}{l}\text { KFL3 Klutuk flowers } \\
\text { extract }(700 \mathrm{mg} / \mathrm{kg} \text { bw })\end{array}$ & $\begin{array}{l}100.0 \pm 4 \\
7\end{array}$ & $\begin{array}{l}168.8 \pm 9.4 \\
\mathrm{ab}\end{array}$ & $\begin{array}{l}145.0 \pm 11.8 \\
\text { abc }\end{array}$ & $\begin{array}{l}122.0 \pm 12.9 \\
\mathrm{abc}\end{array}$ & $\begin{array}{l}110.6 \pm 13.7 \\
\mathrm{bc}\end{array}$ & $136.6 \pm 6.4 \mathrm{abc}$ & $19.1 \pm 5.2$ \\
\hline
\end{tabular}

a: showed significant different compared to normal $(\mathrm{p}<0.01)$; b: showed significant different compared to the negative control $(\mathrm{p}<0.01)$; : showed significant different compared to a positive control $(\mathrm{p}<0.01)$. 
Table 4: Antihyperglycemic effect of peel extracts of $M$. balbisiana by alloxan-induced method

\begin{tabular}{|c|c|c|c|c|c|}
\hline \multicolumn{5}{|c|}{ Blood glucose concentration (mg/dl) } & \multirow{2}{*}{$\begin{array}{l}\text { Blood glucose } \\
\text { reduction }(\%)\end{array}$} \\
\hline Groups & O D (Normal) & $\begin{array}{l}3^{\text {rd }} \text { Day (After alloxan } \\
\text { induced) }\end{array}$ & $\begin{array}{l}1^{\text {th }} \text { Day } \\
\text { (Resolve) }\end{array}$ & $\begin{array}{l}\text { Glucose Concentration } \\
(\mathrm{mg} / \mathrm{dl})\end{array}$ & \\
\hline $\begin{array}{l}\text { Negative control } \\
\text { (CMC-Na } 0,5 \% \text { ) }\end{array}$ & $90.8 \pm 6.38$ & $351.2 \pm 43.02$ & $401.6 \pm 26,76$ & $-50.4 \pm 21.10$ & $-14.98 \pm 7.05$ \\
\hline $\begin{array}{l}\text { Positive control (Glibenclamid } \\
0.65 \mathrm{mg} / \mathrm{kg} \mathrm{bw} \text { ) }\end{array}$ & $9 ., 2 \pm 8.26$ & $345.0 \pm 57.06$ & $120.6 \pm 25,71^{*}$ & $244.4 \pm 44.67$ & $64.87 \pm 5.31$ \\
\hline $\begin{array}{l}\text { KPE1 Klutuk peel extract } \\
(175 \mathrm{mg} / \mathrm{kg} \mathrm{bw})\end{array}$ & $93.6 \pm 4.84$ & $315.8 \pm 62.83$ & $180.8 \pm 45,29 *$ & $135.0 \pm 36.37$ & $42.99 \pm 9.40$ \\
\hline $\begin{array}{l}\text { KPE1 Klutuk peel extract } \\
\text { (350 mg/kg bw) }\end{array}$ & $9 ., 6 \pm 7.09$ & $338.0 \pm 47.70$ & $121.4 \pm 36,98^{*}$ & $216.6 \pm 40.95$ & $64.12 \pm 8.84$ \\
\hline $\begin{array}{l}\text { KPE1 Klutuk peel extract } \\
(700 \mathrm{mg} / \mathrm{kg} \mathrm{bw})\end{array}$ & $90.4 \pm 5.89$ & $345.4 \pm 72.24$ & $132.6 \pm 40,36^{*}$ & $212.8 \pm 44.27$ & $61.81 \pm 5.93$ \\
\hline
\end{tabular}

*:Showed significant different compared to negative control $(\mathrm{p}<0.01)$.

The peel extract of $M$. balbisiana Colla which had the highest reduction percentage of blood glucose level, then was continued by using alloxan-induced method. The results showed that KPE2 gave the highest blood glucose reduction $(64.12 \pm 8.84 \%)$ compared to KPE3 $(61.81 \pm 5.93 \%)$ and KPE $1(42.99 \pm 9.40 \%)$. It was similar to study by Murthy and Felicia which reported that acetone fruit peel extract of M. sapientum $200 \mathrm{mg} / \mathrm{kg}$ bw, which induced by STZ showed the percentage of decreasing of blood glucose level $61.36 \%$ dan $400 \mathrm{mg} / \mathrm{kg}$ bw 63.87\% [25]. Meanwhile, research by Gozali and Mustarichie stated that ethanol extract of ranggap banana (Musa troglodytarum L.) $50 \mathrm{mg} / \mathrm{kg}$ bw could reduce blood glucose level at 4th day (45.97\%), which was induced by alloxan higher than 100 $\mathrm{mg} / \mathrm{kg}$ bw (24.56\%), $150 \mathrm{mg} / \mathrm{kg}$ bw (24.59\%) and $200 \mathrm{mg} / \mathrm{kg}$ bw (18.80\%) [26]. The previous study reported that apiforol compound has been isolated from acetone fraction of $M$. balbisiana Colla seed, which has potential antihyperglycemic activity with IC50 83.54 $\mu \mathrm{g} / \mathrm{ml}$ and $52.87 \mu \mathrm{g} / \mathrm{ml}$ for alfa-amylase and alfa-glucosidase [17]. Borah and Das study demonstrated that flower and stalk of $M$. balbisiana Colla with dose of $250 \mathrm{mg} / \mathrm{kg}$ bw could reduce blood glucose level, which was induced by STZ $30.20 \%$ and $25.58 \%$, respectively [7].

The klutuk peel extract, which gave the highest antihyperglycemic effect was continued by antioxidant activity and performed by DPPH method. The stock solution DPPH $30 \mu \mathrm{g} / \mathrm{ml}$ in methanol was prepared. The method should be verified by using ascorbic acid standard. The klutuk peel extracts $0.5-16 \mu \mathrm{g} / \mathrm{ml}$ were prepared in methanol. By applying calibration curve (percentage of DPPH scavenging activity vs concentration) it can be determined IC50 DPPH of ethanol peel extract was $1.924 \mu \mathrm{g} / \mathrm{ml}$, and it's AAI 7.80. This $\mathrm{AAI}>2$, therefore it can be classified as very strong antioxidant [27]. Meanwhile, ascorbic acid standard had IC50 DPPH $0.179 \mu \mathrm{g} / \mathrm{ml}$ and AAI DPPH 83.80.

The previous results showed that kepok banana peel extract had higher antioxidant activity, which inhibited 50\% oxidation at concentration of $693.15 \mathrm{mg} / \mathrm{ml}$ than ambon banana peel extract at concentration of $5000 \mathrm{mg} / \mathrm{ml}$ [28]. In addition, kepok peel banana extract showed anticancer activity with cytotoxic activity (IC50) $>250 \mu \mathrm{g} / \mathrm{ml}$ [29]. Furthermore, this study showed that IC50 of DPPH of ethanolic peel extracts from white ambon banana was 0.37 $\mu \mathrm{g} / \mathrm{ml}$. Meanwhile, other results showed that ethanolic peel extracts of raja bulu banana, muli banana and ambon lumut banana had IC50 of DPPH scavenging activities were 36.12, 4.39 and $6.91 \mu \mathrm{g} / \mathrm{ml}$, respectively [30]. The other previous study expressed that IC50 of DPPH scavenging activities of ethanolic peel extracts of tanduk banana, nangka banana and kepok banana was 101.40, 53.05 and $6.22 \mu \mathrm{g} / \mathrm{ml}$, respectively [31].

Table 5: Absorbance and percentage of DPPH scavenging activity of ethanolic peel extract of $M$. balbisiana

\begin{tabular}{lll}
\hline Concentration of $\boldsymbol{M}$. balbisiana $(\boldsymbol{\mu g} / \mathbf{m l})$ & Absorbance & DPPH scavenging activity (\%) \\
\hline 0.5 & 0.5021 & 49.19 \\
1 & 0.5001 & 49.39 \\
2 & 0.4939 & 50.02 \\
4 & 0.4804 & 51.39 \\
8 & 0.4695 & 52.49 \\
16 & 0.4411 & 55.36 \\
\hline
\end{tabular}

$\mathrm{IC}_{50} \mathrm{DPPH}=1.9241 \mu \mathrm{g} / \mathrm{ml}, \mathrm{AAI}=7.80$

This tropical fruit have a strong ability to protect themselves from oxidative stress [6], as a weak source of primary antioxidants but a strong source of secondary antioxidants [7, 32]. Antioxidant compounds in bananas were identified such as ascorbic acid, tocopherol, beta-carotene, phenolic groups, dopamine and gallocatechin $[33,34]$. Banana peel is an underused by-product that can be processed to obtain flour that is more easily stored for further uses. The extracts of banana peel flour exhibited a high total phenolic, due to the occurrence of important amounts of flavonoid phenolics, highly polymerized prodelphinidins, followed by lower contents of flavonol glycosides, B-type procyanidin dimers, monomeric flavan-3-ols, and high total phenolic content, which very high antioxidant activity [35]. Flavonoids have activities as antioxidants that can protect the body from damage caused by reactive oxygen species [36]. Furthermore, flavonoids themselves can regenerate pancreatic $\beta$-cells so that insulin deficiency can be overcome [37]. Flavonoids were also expected to reduce blood glucose levels by inhibiting glucose absorption from the gastrointestinal lumen, especially flavonoids which were in the form of glycosides [38].

\section{CONCLUSION}

The ethanolic extract of peel, pulp, seed and flower of klutuk banana had antidiabetic activity. However, the klutuk peel extract (350 $\mathrm{mg} / \mathrm{kg} \mathrm{bw}$ ) gave the highest antihyperglycemic activity than other test groups. The ethanolic klutuk peel extract was very strong antioxidant activity. The klutuk banana peel can be exploited as source of natural anti-diabetes and antioxidant agent.

\section{ACKNOWLEDGEMENT}

The authors thankful to Ministry of education and culture of Indonesia on Penelitian Disertasi Doctoral Grant in 2020, Faculty of Pharmacy of Padjajaran University and Institute of Health Science of Bakti Tunas Husada Tasikmalaya, for supporting the facilities. 


\section{FUNDING}

Nil

\section{AUTHORS CONTRIBUTIONS}

All the authors contributed equally.

\section{CONFLICT OF INTERESTS}

Authors declare no conflict of interest.

\section{REFERENCES}

1. Salam N, Fareed M, Khoja AT, Mahmoud AM, Ahamed M. Life style related risk factors of type 2 diabetes mellitus and its increased prevalence in Saudi Arabia: a brief review utilization of clinical preventive services among Saudi older adults view project lifestyle related risk factors of type 2 diabetes. Int J Med Res Heal Sci 2017;6:125-32.

2. Ligita T, Wicking K, Francis K, Harvey N, Nurjannah I. How people living with diabetes in Indonesia learn about their disease: a grounded theory study. PLoS ONE 2019;14:1-19.

3. Internasional of Diabetic Ferderation (IDF). IDF Diabetes Atlas8th Edition; 2017. Available from: www.diabetesatlas.org. [Last accessed on 15 Aug 2020]

4. Cho NH, Shaw JE, Karuranga S, Huang Y, da Rocha Fernandes JD, Ohlrogge AW, et al. IDF diabetes atlas: global estimates of diabetes prevalence for 2017 and projections for 2045. Diabetes Res Clin Pract 2018;4:271-81.

5. Indonesian Health Ministry. Basic health research of the ministry of health; 2018. p. 1-200.

6. MacBryde, Bruce. Consensus document on the biology of bananas and plantains (Musa sp.) Vol. 43. Paris. OECD Environment Health Safety Publications 2009;43:1-87.

7. Borah M, Das S. Antidiabetic, antihyperlipidemic, and antioxidant activities of Musa balbisiana colla. in type 1 diabetic rats. Indian J Pharmacol 2017;49:71-6.

8. Deka P, Kashyap A, Sharma D, Baruah C. A review on Musa balbisiana colla. Int J Pharm Sci Invent 2018;7:14-7.

9. Tin HS, Padam BS, Kamada T, Vairappan CS, Abdullah MI, Chye FY. Isolation and structure elucidation of triterpenes from inflorescence of banana (Musa balbisiana $c v$. saba). Int Food Res J 2016;23:866-72.

10. Shian TE, Abdullah A, Musa KH, Maskat MY, Ghani MA. Antioxidant properties of three banana cultivars (Musa acuminate berangan, mas and raja) extracts. Sains Malaysiana 2012;41:319-24.

11. Navghare VV, Dhawale SC. In vitro antioxidant, hypoglycemic and oral glucose tolerance test of banana peels. Alexandria Med J 2017;53:237-43.

12. Ighodaro OM, Adeosun AM, Asejeje FO, Soetan GO, Kassim 00. Time course effects of 5,5-dihydroxylpyrimidine-2,4,6-trione (alloxan) as a diabetogenic agent in animal model. Alexandria Med J 2018;54:705-10.

13. Ighodaro OM, Adeosun AM, Akinloye OA. Review alloxaninduced diabetes, a common model for evaluating the glycemiccontrol potential of therapeutic compounds and plants extracts in experimental studies. Medicina 2017;53:365-74.

14. Malpani MO, Rajput PR, Chinchole KV, Kapse SS, Ambarkar KS. Phytochemical screening and antioxidant activity of extracts of Xanthium strumarium, Chrysanthemum and their mixture. Rasayan J Chem 2019;12:1901-8.

15. Tchimene MK, Okoli CO, Iwu MM. Antidiabetic property of some Nigerian medicinal plants. J Med Plants Res 2016;10:139-48.

16. Oskouei BG, Ravasjani SA, Musavinejad SJ, Salehzadeh SA, Abdolhosseinzadeh $\mathrm{A}$, Hamishehkar $\mathrm{H}$, et al. In vivo evaluation of anti-hyperglycemic, anti-hyperlipidemic and antioxidant status of liver and kidney of thymol in STZ-induced diabetic rats. Drug Res (Stuttg) 2019;69:46-52.

17. Gopalan G, Prabha B, Joe A, Reshmitha TR, Sherin DR, Abraham B, et al. Screening of Musa balbisiana colla. seeds for antidiabetic properties and isolation of apiforol, a potential lead, with antidiabetic activity. J Sci Food Agric 2019;99:2521-9.

18. Bisht AJ, Juyal D, Monika, Verma S, Joshi S. Study on in vitro antidiabetic potential of stem part of Musa paradisiaca linn. Antimicrobial and phytochemical analysis of leaf extract of medicinal fruit plants. Int J Compr Adv Pharmacol 2017;2:1-5.

19. Verma S, Sahdev AK, Raj V. Stem of Musa paradisiaca (linn.) shows the inhibitory effect towards alpha-amylase and alphaglucosidase enzymes: antidiabetic activity. Adv Tissue Eng Regen Med 2017;2:164-6.

20. Nguyen D, Novakova A, Spurna K, Hricko J, Phung H, Viktorova $\mathrm{J}$, et al. Antidiabetic compounds in stem juice from banana. Czech J Food Sci 2017;35:7-13.

21. Borborah K, Borthakur SK, Tant B. Musa balbisiana Colla taxonomy, traditional knowledge and economic potentialities of the plant in Assam, India. Indian J Trad Knowledge 2016;15:116-20.

22. Jannat K, Morshed Md Z, Akter S, Rahmatullah M. Improved oral glucose tolerance with ripe fruit peels of Musa seminifera lour. Arch Nat Med Chem 2018;13:1-4.

23. Genatrika E, Laksari VNH, Tjiptasurasa. Antidiabetic activity of Musa acuminata colla fruit peel (MACFP) ethanol extract in glucose-induced diabetic rats. MATEC Web Conf 2018;197:1-4.

24. Al-Mahamud R, Jannat $\mathrm{K}$, Islam M, Shova NA, Jahan R, Hossain Md N, et al. Variations in oral glucose tolerance is present in different sub-cultivars of fruit skins of Musa sapientum L (banana). World J Pharm Res 2018;7:192-9.

25. Murthy SSN, Felicia C. Antidiabetic activity of Musa sapientum fruit peel on STZ induced diabetic rats. Int J Pharm Bio Sci 2015;6:537-43.

26. Gozali D, Mustarichie R. Antidiabetic activity of ethanol extract of ranggap bananas Musa troglodytarum L. Int Res J Pharm 2018;9:80-4.

27. Scherer R, Godoy HT. Antioxidant activity index (AAI) by the 2,2-diphenyl-1-picrylhydrazyl method. Food Chem 2009;112:654-8.

28. Rosdiana R. Fortification of tofu using antioxidants from kepok banana peel extract (Musa bluggoe). repository.upi.edu; 2014.

29. Wardati F, Holil K, Syarifah U. Cytotoxicity test on breast cancer cell lines T-47D treated with pisang kepok peel extract (Musa balbisiana). J Trop Life Sci 2018;1:84-8.

30. Fidrianny I, Rizki K, Insanu M. In vitro antioxidant activities from various extracts of banana peels using ABTS, DPPH assays and correlation with phenolic, flavonoid, carotenoid content. Int J Pharm Pharm Sci 2014;6:299-303.

31. Fidrianny I, Sefiany E, Ruslan K. In vitro antioxidant activities from three organs of white ambon banana (Musa AAA Group) and flavonoid, phenolic, carotenoid content. Int J Pharmacogn Phytochem Res 2015;7:590-6.

32. Haripyaree A, Guuneshwor K, Damayanti M. Evaluation of antioxidant properties of some wild edible fruits extracts by cell-free assays. Elec J Env Agricult Food Chem 2010;9:345-450.

33. Yin X, Quan J, Kanazawa T. Banana prevents plasma oxidative stress in healthy individuals. Plant Foods Hum Nutr 2008;63:71-6.

34. Qusti SY, Abo Khatwa AN, Lahwa MA. Free radical scavenger enzymes of fruit plant species cited in Holy Quran. World Appl Sci J 2010;9:338-44.

35. Saritha M. Flavonoids-the most potent poly-phenols as antidiabetic agents: an overview. Mod Appro Drug Des 2017;1:1-5.

36. Rebelloa LPG, Ramosb AM, Pertuzattic PB, Barciad MT, Munoze NC, Gutierrezf IH. Flour of banana (Musa AAA) peel as a source of antioxidant phenolic compounds. Food Res Int 2014;55:397-403.

37. Marianne, Yuandani, Rosnani. Antidiabetic activity from ethanol extract of Kluwih's leaf (Artocarpus camansi). J Natural 2011;11:64-8.

38. Vijayakumar S, Presannakumar G, Vijayalakshmi NR Investigations on the effect of flavonoids from banana, Musa paradisiaca L. on lipid metabolism in rats. J Diet Suppl 2009;6:111-23. 